\title{
Back to the start: Evaluation of prognostic markers in gastrointestinal stromal tumors
}

\author{
ECKHARD KLIESER $^{1 *}$, MAXIMILIAN PICHELSTORFER $^{1,2^{*}}$, DENIS WEYLAND $^{1,2}$, \\ RALF KEMMERLING ${ }^{1}$, STEFAN SWIERCZYNSKI ${ }^{3}$, ADAM DINNEWITZER ${ }^{3}$, \\ TARKAN JÄGER ${ }^{3}$, TOBIAS KIESSLICH ${ }^{4,5}$, DANIEL NEUREITER $^{1}$ and ROMANA ILLIG ${ }^{1}$ \\ ${ }^{1}$ Institute of Pathology, Paracelsus Medical University/Salzburg General Hospital (SALK), A-5020 Salzburg; \\ ${ }^{2}$ Bio- and Environmental Technology Program, University of Applied Sciences Upper Austria, A-4600 Wels; \\ Departments of ${ }^{3}$ Surgery and ${ }^{4}$ Internal Medicine I, Paracelsus Medical University/Salzburg General Hospital (SALK); \\ ${ }^{5}$ Laboratory for Tumor Biology and Experimental Therapies (TREAT), Institute of Physiology \\ and Pathophysiology, Paracelsus Medical University, A-5020 Salzburg, Austria
}

Received December 9, 2015; Accepted February 26, 2016

DOI: $10.3892 / \operatorname{mco} .2016 .819$

\begin{abstract}
The aim of this study was to provide a standardized risk stratification model for gastrointestinal stromal tumors (GISTs) based on tumor localization, tumor size, involved lymph nodes and metastases, as well as mitotic activity and other morphological and molecular markers, in order to improve the risk evaluation scheme for recurrence, metastatic spread and survival for patients with GIST. A total of 201 cases of patients with GIST were investigated according to standardized morphological markers, including nuclear pleomorphism, tumor cell necrosis, mucosal infiltration, ulceration, skeinoid fibers and growth pattern. In addition, all cases were immunohistochemically analyzed using a tissue microarray platform for various markers of differentiation (CD34, CD44, CD117, desmin, discovered on GIST 1, platelet-derived growth factor receptor $\alpha$, S-100 and smooth muscle actin) and proliferation (B-cell lymphoma 2, P16, P53, phosphohistone H3 and Ki-67). These findings were
\end{abstract}

Correspondence to: Dr Daniel Neureiter, Institute of Pathology, Paracelsus Medical University/Salzburg General Hospital (SALK), 48 Müllner Hauptstrasse, A-5020 Salzburg, Austria

E-mail:d.neureiter@salk.at

*Contributed equally

Abbreviations: ANOVA, analysis of variance; Bcl-2, B-cell lymphoma 2; CI, confidence interval; DOG-1, discovered on GIST 1; FFPE, formalin-fixed and paraffin-embedded; GIST, gastrointestinal stromal tumor; GP, growth pattern; H\&E, hematoxylin and eosin; HPF, high-power field; IHC, immunohistochemistry; MI, mucosal infiltration; MU, mucosal ulceration; NP, nuclear pleomorphism; OD, odds ratio; PDGFR $\alpha$, platelet-derived growth factor receptor $\alpha$; PHH3, phosphohistone H3; SF, skeinoid fibers; SMA, smooth muscle actin; TCN, tumor cell necrosis; TMA, tissue microarray

Key words: gastrointestinal stromal tumors, prognostic markers correlated by uni- and multivariable analyses with clinicopathological characteristics, including recurrence, metastasis and survival. The general clinicopathological parameters of this GIST specimen cohort were comparable to previous studies. While several parameters exhibited clear associations to each other and to the defined clinical endpoints, the multivariate analysis reduced the number of relevant prognostic variables to localization, margin status, growth pattern and hematoxylin and eosin-based mitosis/Ki-67-based proliferation of GISTs. With the exception of CD34, none of the applied markers of differentiation and proliferation were found to be independent prognostic markers in GIST and the classical risk factors of GIST remain important prognostic factors. Additionally, growth pattern may predict the risk of recurrence and metastasis in GIST patients. Additional independent molecular prognostic markers remain to be identified and validated.

\section{Introduction}

With an incidence of 1.4-2 cases per 100,000/year, gastrointestinal stromal tumors (GISTs) are the most frequent mesenchymal gastrointestinal neoplasms (1). GISTs mainly affect individuals aged $>50$ years and are more likely localized in the stomach $(60 \%)$ compared with the jejunum and ileum (30\%), duodenum (4-5\%), rectum (4\%), remaining colon and appendix $(1-2 \%)$ and esophagus $(<1 \%)$. Approximately $30-50 \%$ of GISTs are malignant and, in $\sim 50 \%$ of the cases, metastasis has occurred prior to the initial diagnosis (2). Morphologically, GISTs are classified into spindle-cell (70\%), epitheloid (20\%) or mixed types (10\%) (3). The most common symptoms of GISTs are anemia, weight loss, gastrointestinal bleeding, abdominal pain or tumor mass-related symptoms. Complete surgical resection is the main goal of therapy. A tyrosine kinase inhibitor, imatinib (STI-571), was recently approved for the (neo)adjuvant treatment of GISTs (4).

Malignant GIST lesions are more common compared with benign tumors in the intestine, whereas the opposite is true in the stomach. The main factors for risk stratification 
are tumor size, mitotic rate and anatomical location. Tumors sized $<2.0 \mathrm{~cm}$ with low mitotic activity $[<5$ mitoses per 50 high-power fields (HPFs)] are usually benign, whereas tumors exhibiting $>5$ mitoses per 50 HPFs are likely to be malignant (5). This rule is applied for the classification of malignant GISTs, although there are exceptions for tumors which are considered to be benign by the accepted criteria, as lesions sized $<2.0 \mathrm{~cm}$ and/or mitotic rate $<5 / 50 \mathrm{HPFs}$ occasionally metastasize, the reason for that remains unclear (3).

There are different risk stratification systems to classify GISTs from low- to high-risk, using parameters such as the mindbomb E3 ubiquitin protein ligase 1 index, histological subtype, alterations related to specific oncoproteins, such as P16, unfavorable kinase mutations or cytogenetic aberrations. The problem is that the different classes of GISTs are reported to overlap in a number of cases $(6,7)$. For this reason, additional factors are required to reliably predict the behaviour of these tumors (8). These additional factors may be morphological as well as molecular markers, verified by means of immunohistochemistry (IHC).

The aim of the present study was to analyze different morphological and molecular biological markers in GISTs, using well-defined morphological criteria in hematoxylin and eosin (H\&E) as well as immunohistochemical stainings based on tissue microarray platform, in order to identify further possible determining factors for recurrence, metastasis and survival.

\section{Materials and methods}

Patient and sample characteristics. The present study included 201 formalin-fixed and paraffin-embedded (FFPE) tissue samples of primary GIST specimens resected between 1997 and 2014 [115 (57.2\%) from female and $86(42.8 \%)$ from male patients], with complete histopathological records (spindle-shaped, epitheloid or mixed histomorphological subtypes) and tumor classification according to the TNM staging system (9). The general characteristics of the investigated GISTs are summarized in Table I. The range of fixation time for the obtained specimens was determined between 12 and $24 \mathrm{~h}$ in $4 \%$ phosphate-buffered saline-formalin solution to avoid false-positive or -negative immunohistochemical staining patterns due to under- or over-fixation $(10,11)$.

Morphology. A formally well-defined list of histomorphological criteria $(7,12)$ was used to retrospectively investigate all GIST cases in terms of nuclear pleomorphism (NP; no, low, moderate, or severe), tumor cell necrosis (TCN; absence or presence), mucosal infiltration (MI; absence or presence), mucosal ulceration (MU; absence or presence), skeinoid fibers (SF; absence or presence) and growth pattern (GP; type I-luminal/intramural or type II-extramural).

Based on 5- $\mu \mathrm{m} \mathrm{H \& E-stained} \mathrm{FFPE} \mathrm{sections,} \mathrm{mitotic}$ figures were counted in 50 consecutive HPFs as previously described $(3,9)$ under a Leica DM2000 microscope (Leica Microsystems, Vienna, Austria) by two independent investigators (RK and DN).

IHC. IHC was routinely performed on an Autostainer Plus (Dako Austria GmbH, Vienna, Austria), according to the manufacturer's recommendations (13), using heat-induced epitope retrieval in $\mathrm{pH} 9.0$ antigen retrieval buffer (Dako Austria $\mathrm{GmbH}$ ) at $95^{\circ} \mathrm{C}$ for $40 \mathrm{~min}$ for the applied antibodies (Table II).

Immunohistochemical stainings were performed on whole slides for phosphohistone $\mathrm{H} 3$ (PHH3) and $\mathrm{Ki}-67$, whereas all other immunohistochemical stainings were performed on tissue microarrays (TMAs), as previously described in detail (14). In brief, TMAs were prepared from paraffin-embedded tissues with a designed grid of $2.0-\mathrm{mm}$ black circles leaving a $2.0-\mathrm{mm}$ space between them on plain paper fixed to stainless steel moulds. Dried cores of paraffin-embedded GIST tissue blocks obtained by a $2.0-\mathrm{mm}$ Harris Uni-Core punch (Ted Pella Inc., Redding, CA, USA) were aligned to the grid. Once all cores were attached to the mould, melted paraffin was gently poured into the mould and the TMA was handled according to routine histopathological procedures from this point onwards.

Interpretation of $\mathrm{IHC}$. $\mathrm{PHH} 3$-positive cells were manually counted in 50 HPFs. The Ki-67-based proliferation rate was assessed by the optimized particle analysis module according to the software manual (Image Access 9 Enterprise; Imagic Bildverarbeitung AG, Glattbrugg, Switzerland) on three digitized hot spot areas and related to the total number of cells, as previously described (15).

All other immunohistochemical stainings were evaluated using a modified quickscore method by multiplication of the intensity [rated as 0 (negative), 1 (low), 2 (moderate) or 3 (strong)] and extent of positive cells (0-100\%), which yields values ranging from 0 (negative) to a maximum of 300 (staining intensity of 3 and positive cells 100\%) (16). Image evaluation was independently performed by two investigators (MP and DN). Differences in assessment were discussed until consensus was reached.

Ethics. This study was conducted following our National and Institutional guidelines and in accordance with the Declaration of Helsinki (1964); the samples are exclusively available for research purposes in retrospective studies. Based on the retrospective nature of this study and full anonymization of the patient data, the present study is not subject to formal approval by the appropriate local Ethics Committee (http://www.salzburg.gv.at/ethikkommission).

Statistical analysis. Statistical analysis was performed with IBM SPSS 20.0 software (IBM Corp., Armonk, NY, USA). The $\chi^{2}$-test or Student's t-test was used to compare data of nominal or interval level. The Student's t-test and univariate analysis of variance (ANOVA) using Bonferroni's post-hoc test to adjust for multiple comparisons were applied for differences between two and more groups of tissue samples, respectively. For survival analysis, cases with missing date of recurrence, metastasis or death were excluded. Multivariate analysis was performed using time-independent binary logistic regression or time-dependent Cox-regression technique for recurrence, metastasis or death. Based on the predicted probabilities of the regression analysis, dichotomous variables were calculated with optimized cluster analysis for additional survival analysis using the Kaplan-Meier method comparing the curves of recurrence, metastasis and 


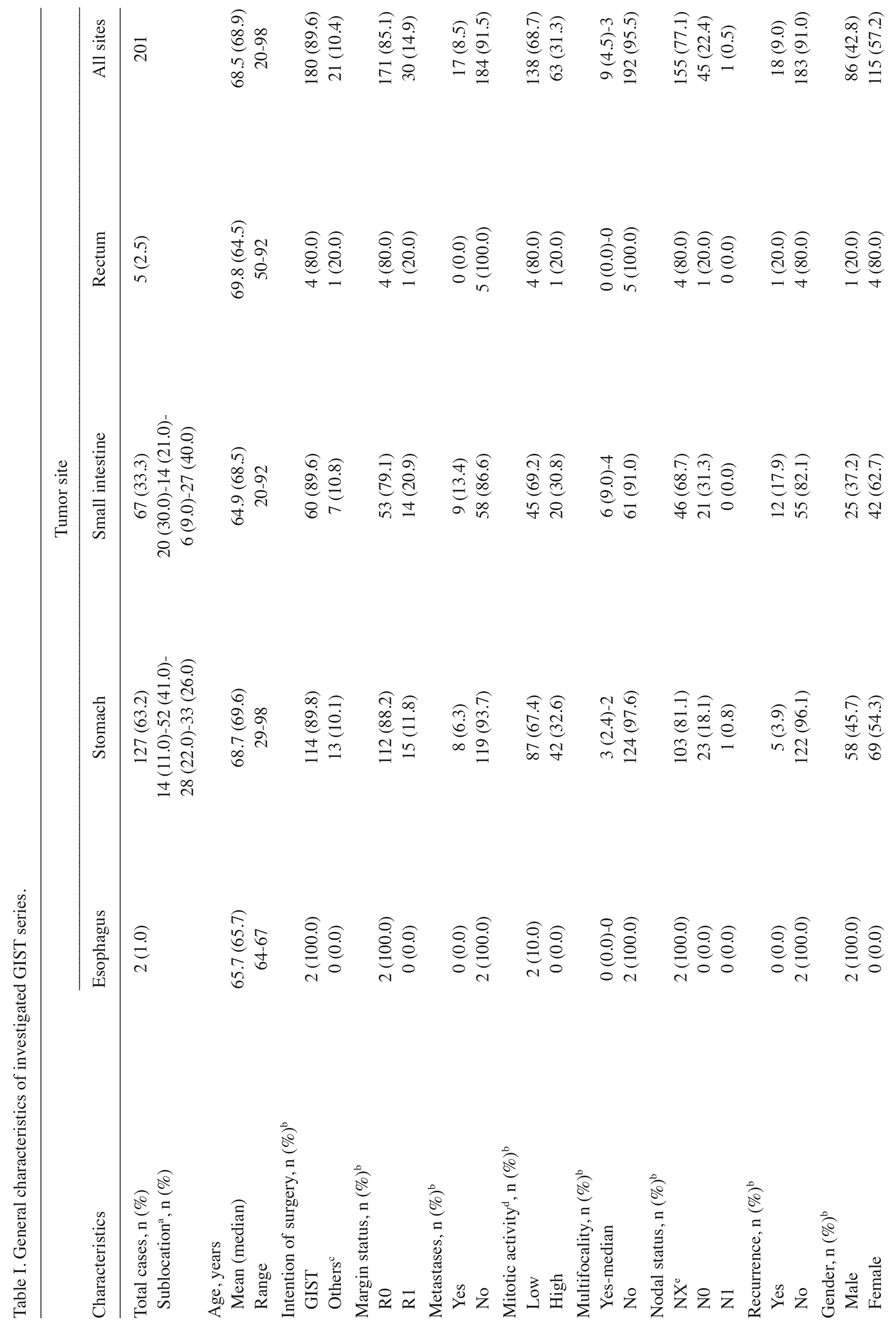




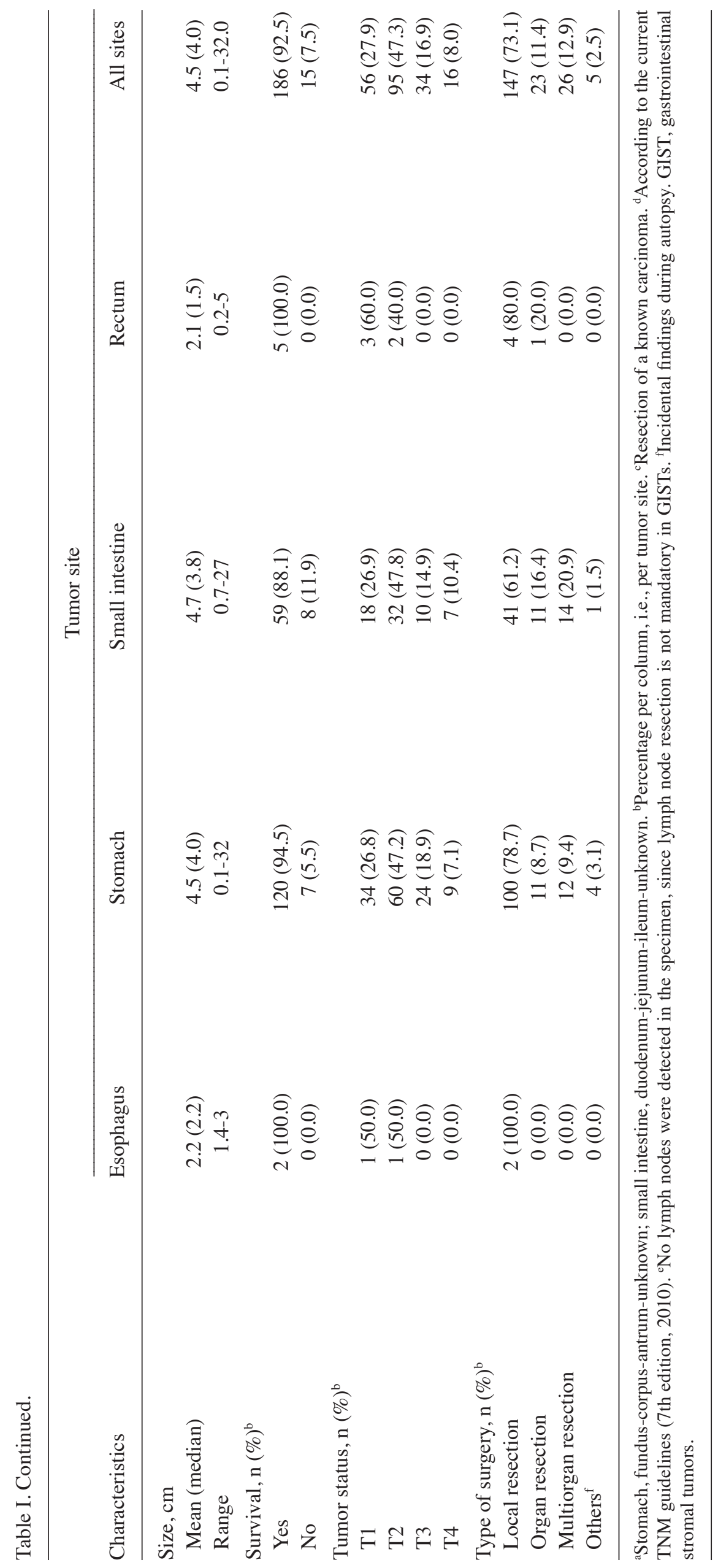


Table II. Antibodies and conditions used for immunohistochemistry.

\begin{tabular}{|c|c|c|c|c|c|}
\hline Antibody & Catalogue no. & Clone & Species & Dilution/incubation & Pretreatment ${ }^{\mathrm{a}}$ \\
\hline Bcl-2 & M0887 & 124 & Mouse & $1: 100 / 30 \mathrm{~min}$ & $\mathrm{WB} / \mathrm{pH} 9$ \\
\hline CD34 & IR632 ${ }^{b}$ & QB END10 & Mouse & Ready to use/30 min & $\mathrm{WB} / \mathrm{pH} 9$ \\
\hline CD44 & NCL-CD44-2 ${ }^{c}$ & DF 1485 & Mouse & $1: 100 / 30 \mathrm{~min}$ & $\mathrm{WB} / \mathrm{pH} 9$ \\
\hline CD117 (C-kit) & $\mathrm{A} 4502^{\mathrm{b}}$ & C-kit & Rabbit & $1: 200 / 30 \mathrm{~min}$ & $\mathrm{WB} / \mathrm{pH} 9$ \\
\hline Desmin & IR606 ${ }^{\mathrm{b}}$ & D33 & Mouse & Ready to use/30 min & $\mathrm{WB} / \mathrm{pH} 9$ \\
\hline DOG-1 & NCL-L-DOG-1 ${ }^{\mathrm{c}}$ & K9 & Mouse & $1: 100 / 30 \mathrm{~min}$ & $\mathrm{WB} / \mathrm{pH} 9$ \\
\hline Ki67 & M7240 & MIB-1 & Mouse & $1: 500 / 30 \mathrm{~min}$ & $\mathrm{WB} / \mathrm{pH} 9$ \\
\hline P16 & $7254713^{\mathrm{d}}$ & EGH4 & Mouse & Ready to use/30 min & $\mathrm{pH} 9$ \\
\hline P53 & $\mathrm{M} 7001^{\mathrm{b}}$ & DO7 & Mouse & $1: 200 / 30 \mathrm{~min}$ & $\mathrm{WB} / \mathrm{pH} 9$ \\
\hline $\operatorname{PDGFR} \alpha$ & RB-9027-P1 ${ }^{\mathrm{e}}$ & Not reported & Rabbit & $1: 50 / 30 \mathrm{~min}$ & pH 9 \\
\hline РHH3 & $369 A-18^{f}$ & Not reported & Rabbit & Ready to use/30 min & $\mathrm{WB} / \mathrm{pH} 9$ \\
\hline S-100 & $\mathrm{Z} 0311^{\mathrm{b}}$ & Not reported & Rabbit & $1: 2000 / 30 \mathrm{~min}$ & $\mathrm{WB} / \mathrm{pH} 6$ \\
\hline SMA & M0851 ${ }^{\mathrm{b}}$ & $1 \mathrm{~A} 4$ & Mouse & $1: 600 / 30 \mathrm{~min}$ & $\mathrm{WB} / \mathrm{pH} 9$ \\
\hline
\end{tabular}

${ }^{\mathrm{a}} \mathrm{pH} 6$ or 9, heat-induced epitope retrieval in $\mathrm{pH} 6$ or 9 antigen retrieval buffer (Dako,Glostrup, Denmark) and WB in a Pascal pressurized heating chamber (Dako). Purchased from ${ }^{\mathrm{b}}$ Dako; ${ }^{\mathrm{C}}$ Novocastra/Leica Microsystems (Vienna, Austria); ${ }^{\mathrm{d}}$ Ventana (Tucson, AZ, USA); ${ }^{\mathrm{e}}$ Thermo Scientific (Waltham, MA, USA) and ${ }^{\mathrm{f} C e l l ~ M o r q u e ~(R o c k l i n, ~ C A, ~ U S A) . ~ B c l-2, ~ B-c e l l ~ l y m p h o m a ~ 2 ; ~ D O G-1, ~ d i s c o v e r e d ~ o n ~ G I S T ~ 1 ; ~ P D G F R ~} \alpha$, platelet-derived growth factor receptor $\alpha$; PHH3, phosphohistone H3; SMA, smooth muscle actin; WB, waterbath; GIST, gastrointestinal stromal tumor; MIB-1, mindbomb E3 ubiquitin protein ligase 1.

survival with the log-rank test. For all calculations, $\mathrm{P}<0.05$ and $\mathrm{P}<0.01$ were considered to indicate statistically significant or highly significant differences, respectively.

\section{Results}

Major clinicopathological, morphological and immunohistochemical characteristics in GISTs

Clinicopathological characteristics (Table I). Overall, 201 patients [115 women (57.2\%) and 86 men (42.8\%); median age at the time of diagnosis, 68.9 years] were included in the present study. The most common localization of GISTs was the stomach $(n=127)$ followed by the small intestine $(n=67)$, the rectum $(n=5)$ and the esophagus $(\mathrm{n}=2)$, with a median tumor size of $4.0 \mathrm{~cm}$ (range, 0.1-32.0 cm), corresponding to the main tumor status of pT2, according to the current TNM guidelines (9), whereas the more locally advanced GISTs (pT3 and pT4) were limited to the stomach and the small intestine. Only 1 case $(0.5 \%)$ exhibited lymph node metastasis, whereas the lymph node detection rate was overall very low $[n=46(22.9 \%)]$. Multifocality $[n=9(4.5 \%)]$ was observed in the small intestine $(n=6)$ and the stomach $(n=3)$. The intention of surgery was mainly due to preoperatively diagnosed or suspected GIST [n=180 (89.6\%)], or incidentally discovered in the remaining cases $[n=21(10.4 \%)]$. Local organ-sparing GIST resection was the most frequently selected type of surgery [ $\mathrm{n}=147$ (73.1\%)], with an incomplete resection (R1) in 30 cases (14.9\%), mostly observed in the small intestine (20.9\%). Recurrence of the GIST was observed in 18 cases (9.0\%; mean/median, 35.4/24.6 months) and metastasis in 17 cases $(8.5 \%$; mean/median, 45.0/41.7 months). Finally, to date, 15 patients (7.5\%; mean/median, 49.6/38.5 months) included in this study have succumbed to the disease. The cut-off date of the events was July 1st, 2015.
The statistical analysis revealed that the localization of the GISTs, resection status, tumor status (according to TNM), tumor size and H\&E-based grading are significantly associated with recurrence of GISTs. Furthermore, tumor size and H\&E-based grading are significantly associated with metastasis of GISTs $\left(\mathrm{P}<0.05, \chi^{2}\right.$ and t-test). Finally, a significant association was observed between intention and type of surgery, as well as between multifocality and survival $(\mathrm{P}<0.05$, $\chi^{2}$ test), whereas all other clinicopathological characteristics did not affect recurrence, metastasis or patient survival.

Histomorphological characteristics (Table III). As regards histomorphological pattern, spindle-cell morphology was predominantly observed $(61.2 \%)$ compared with the epitheloid and mixed cell types. The median number of mitoses of 3 per 50 HPFs revealed that more cases of GISTs are of low mitotic rate according to the current TNM guidelines, whereby the mitosis count varies widely ( $\leq 266$ per $50 \mathrm{HPFs}$ ), reflecting the mitotic heterogeneity of GISTs. Notably, NP was low, MI and MU were detectable in approximately one-third of all cases, whereas TCN and SF were most frequently found in GISTs. Finally, the luminal/intramural GP was predominant in this cohort of GISTs.

Comparing the different GIST sub-localizations, significant differences in grade of NP ( $\mathrm{P}=0.044$, ANOVA), TCN, MI and luminal/intramural GP $\left(\mathrm{P}<0.05, \chi^{2}\right.$-test $)$ were observed in the small intestine.

Immunohistochemical findings (Table IV). Based on the applied quickscore method by multiplication of the intensity and extent of the staining, the obtained scores for markers of differentiation (in increasing order, S-100 $<$ desmin $<$ SMA $<<<$ PDGFR $\alpha$ $<<$ DOG $-1<$ CD34<CD117) were significantly higher compared with those of cell proliferation/apoptosis markers (in increasing order, $\mathrm{P} 53<\mathrm{P} 16<<\mathrm{CD} 44<\mathrm{Bcl}-2)$. 
Table III. Histomorphological characteristics of the GIST series.

Tumor site

\begin{tabular}{|c|c|c|c|c|c|}
\hline \multirow[b]{2}{*}{ Characteristics } & \\
\hline & Esophagus & Stomach & Small intestine & Rectum & All sites \\
\hline \multicolumn{6}{|c|}{ Growth pattern ${ }^{\mathrm{a}, \mathrm{b}}, \mathrm{n}(\%)^{\mathrm{c}}$} \\
\hline Luminal/intramural & $2(100.0)$ & $107(84.3)$ & $46(68.7)$ & $5(100.0)$ & $160(79.6)$ \\
\hline Extramural & $0(100.0)$ & $20(15.7)$ & $21(31.3)$ & $0(100.0)$ & $41(20.4)$ \\
\hline \multicolumn{6}{|c|}{ Histomorphological pattern, $\mathrm{n}(\%)^{\mathrm{c}}$} \\
\hline Spindle-cell & $2(100.0)$ & $72(56.7)$ & $45(67.2)$ & $4(80.0)$ & $123(61.2)$ \\
\hline Epitheloid & $0(0.0)$ & $29(22.8)$ & $9(13.4)$ & $1(20.0)$ & $39(19.4)$ \\
\hline Mixed type & $0(0.0)$ & $26(20.5)$ & $13(19.4)$ & $0(0.0)$ & $39(19.4)$ \\
\hline \multicolumn{6}{|c|}{ Mitoses/50 HPFs, $\mathrm{n}(\%)^{\mathrm{c}}$} \\
\hline Median (range) & $1(1-1)$ & $3(0-266)$ & $3(0-117)$ & $2(0-6)$ & $3(0-266)$ \\
\hline Mean \pm SD & $1.00 \pm 1.0$ & $7.83 \pm 24.10$ & $12.76 \pm 23.6$ & $2.60 \pm 2.4$ & $9.27 \pm 23.6$ \\
\hline \multicolumn{6}{|c|}{ Mucosal infiltration $^{\mathrm{b}}, \mathrm{n}(\%)^{\mathrm{c}}$} \\
\hline Yes & $2(100.0)$ & $85(66.9)$ & $34(50.7)$ & $5(100.0)$ & $126(62.7)$ \\
\hline No & $0(0.0)$ & $42(33.1)$ & $33(49.3)$ & $0(0.0)$ & $75(37.3)$ \\
\hline \multicolumn{6}{|c|}{ Mucosal ulceration, $\mathrm{n}(\%)^{\mathrm{c}}$} \\
\hline Yes & $2(100.0)$ & $87(68.5)$ & $43(64.2)$ & $4(80.0)$ & $136(67.7)$ \\
\hline No & $0(0.0)$ & $40(31.5)$ & $24(35.8)$ & $1(20.0)$ & $65(32.3)$ \\
\hline \multicolumn{6}{|c|}{ Nuclear pleomorphism ${ }^{\mathrm{b}, \mathrm{d}}$} \\
\hline Median (range) & $0.5(0-1.0)$ & $1.0(0-2,5)$ & $1.5(0-2,5)$ & $1.0(0-1,5)$ & $1.0(0-2,5)$ \\
\hline Mean \pm SD & $0.50 \pm 0.707$ & $1.20 \pm 0.543$ & $1.33 \pm 0.519$ & $0.90 \pm 0.548$ & $1.23 \pm 0.543$ \\
\hline \multicolumn{6}{|l|}{ Skeinoid fibers, $\mathrm{n}(\%)^{\mathrm{c}}$} \\
\hline Yes & $1(50.0)$ & $56(44.1)$ & $39(58.2)$ & $2(40.0)$ & $98(48.8)$ \\
\hline No & $1(50.0)$ & $71(55.9)$ & $28(41.8)$ & $3(60.0)$ & $103(51.2)$ \\
\hline \multicolumn{6}{|c|}{ Tumor cell necrosis $^{\mathrm{b}}, \mathrm{n}(\%)^{\mathrm{c}}$} \\
\hline Yes & $2(100.0)$ & $42(33.1)$ & $22(32.8)$ & $4(80.0)$ & $70(34.8)$ \\
\hline No & $0(0.0)$ & $85(66.9)$ & $45(67.2)$ & $1(20.0)$ & $131(65.2)$ \\
\hline
\end{tabular}

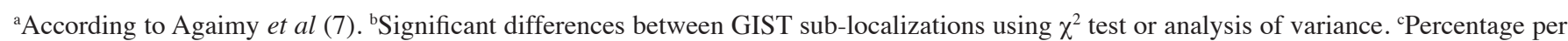
column, i.e., per tumor site. ${ }^{\mathrm{d}} 0$, no; 1 , low; 2, moderate; and 3, strong. GIST, gastrointestinal stromal tumor; HPFs, high-power fields; SD, standard deviation.

Comparing the different GIST sub-localizations, a significant correlation between localization and expression of SMA, C-kit, DOG-1, desmin, CD34 and Bcl-2 were observed $(\mathrm{P}<0.01$, ANOVA).

Univariate statistical analysis of association between clinicopathological, histomorphological and immunohistochemical characteristics

Histomorphological vs. clinicopathological characteristics. The univariate analysis revealed that the majority of the morphological findings were significantly associated with low or high mitotic activity (NP, TCN, MI, MU and SF) $(\mathrm{P}<0.05$, $\chi^{2}$ test) and pT-stage (NP, TCN, MI, MU and GP) $(\mathrm{P}<0.01$, $\chi^{2}$ test), whereby only multifocality and resection status were significantly associated with the GIST GP $\left(\mathrm{P}<0.01, \chi^{2}\right.$ test).

Compared with the events of recurrence, metastasis and survival, NP, TCN and GP exhibited a significant association with recurrence and metastasis $\left(\mathrm{P}<0.05, \chi^{2}\right.$ test $)$, whereas no morphological characteristics were associated with survival.
Immunohistochemical vs. clinicopathological characteristics. The statistical analysis revealed that $\mathrm{Ki}-67-$ based proliferation rate was significantly associated with all the clinicopathological findings, whereas only certain markers of differentiation (S-100, DOG-1, CD34, CD44 and desmin) and proliferation (P16, P53 and Bcl-2) were significantly associated clinicopathological parameters, such as size (S-100, DOG-1, CD34, CD44 and Bcl-2), multifocality (DOG-1), grading (PHH3, CD-117, desmin, CD44 and Bcl-2) and tumor stage (desmin, P16, P53 and Bcl-2) using t-test or ANOVA $(\mathrm{P}<0.05)$.

As regards recurrence, metastasis and survival status, Ki-67, CD117 and CD34 scores were significantly associated with events of recurrence, and Ki-67 and CD117 scores were associated with detected metastases. Finally, Ki-67, CD34 and PDGFR $\alpha$ scores were significantly different in surviving and non-surviving patients $(\mathrm{P}<0.05$, t-test).

Histomorphological vs. immunohistochemical characteristics. Heterogeneous sets of immunohistochemical factors are statistically associated with NP (Ki-67, PHH3, S-100, 
Table IV. Immunohistochemical characteristics of the GIST series.

\begin{tabular}{|c|c|c|c|c|c|}
\hline \multirow[b]{2}{*}{ Characteristics } & \multicolumn{5}{|c|}{ Tumor site } \\
\hline & Esophagus & Stomach & Small intestine & Rectum & All sites \\
\hline \multicolumn{6}{|l|}{$\mathrm{Bcl}-2^{\mathrm{a}}$} \\
\hline I & $0.5(0.5)$ & $1.9(2.0)$ & $2.6(3.0)$ & $1.8(1.5)$ & $2.1(2.0)$ \\
\hline E & $5(5.0)$ & $70(80.0)$ & $86(90.0)$ & $58(60.0)$ & $74(90)$ \\
\hline $\mathrm{S}$ & $5 \pm 7$ & $158 \pm 98$ & $236 \pm 84$ & $123 \pm 117$ & $178 \pm 102$ \\
\hline \multicolumn{6}{|l|}{ CD117 (C-kit) ${ }^{\mathrm{a}}$} \\
\hline I & $1.8(1.8)$ & $2.0(2.0)$ & $2.5(3.0)$ & $2.6(2.8)$ & $2.2(2.0)$ \\
\hline $\mathrm{E}$ & $58(57.5)$ & $79(95.0)$ & $93(100.0)$ & 99 (100.0) & $84(100.0)$ \\
\hline S & $109 \pm 101$ & $183 \pm 101$ & $236 \pm 89$ & $260 \pm 49$ & $202 \pm 99$ \\
\hline \multicolumn{6}{|l|}{$\mathrm{CD} 34^{\mathrm{a}}$} \\
\hline I & $1.8(1.8)$ & $2.4(3.0)$ & $1.7(2.0)$ & $2.5(2.5)$ & $2.2(2.5)$ \\
\hline E & $50(50.0)$ & $81(100.0)$ & $52(45.0)$ & $96(97.5)$ & $72(95.0)$ \\
\hline $\mathrm{S}$ & $95 \pm 92$ & $220 \pm 106$ & $126 \pm 117$ & $239 \pm 45$ & $189 \pm 117$ \\
\hline \multicolumn{6}{|l|}{ CD44 } \\
\hline I & $0.5(0.5)$ & $0.7(1.0)$ & $1.0(1.0)$ & $0.5(0.5)$ & $0.8(1.0)$ \\
\hline $\mathrm{E}$ & $3(2.5)$ & $25(10.0)$ & $34(30.0)$ & $13(10.0)$ & $27(20.0)$ \\
\hline S & $3 \pm 4$ & $39 \pm 64$ & $62 \pm 92$ & $13 \pm 15$ & $46 \pm 75$ \\
\hline \multicolumn{6}{|l|}{$\operatorname{Desmin}^{\mathrm{a}}$} \\
\hline I & $1.0(1.0)$ & $0.3(0.0)$ & $0.2(0.0)$ & $0.4(0.0)$ & $0.3(0.0)$ \\
\hline E & $48(47.5)$ & $4(0.0)$ & $3(0.0)$ & $2(0.0)$ & $4(0.0)$ \\
\hline S & $95 \pm 134$ & $6 \pm 19$ & $7 \pm 37$ & $2 \pm 3$ & $7 \pm 29$ \\
\hline \multicolumn{6}{|l|}{ DOG- $1^{2}$} \\
\hline I & $1.5(1.5)$ & $1.6(1.5)$ & $2.2(2.0)$ & $2.5(2.5)$ & $1.8(2.0)$ \\
\hline $\mathrm{E}$ & $48(47.5)$ & $70(80.0)$ & $86(100.0)$ & $91(97.5)$ & $75(90.0)$ \\
\hline S & $87 \pm 103$ & $133 \pm 92$ & $209 \pm 94$ & $231 \pm 75$ & $160 \pm 99$ \\
\hline \multicolumn{6}{|l|}{$\mathrm{Ki}-67\left(/ \mathrm{mm}^{2}\right)^{\mathrm{b}}$} \\
\hline Median (range) & $47.2(44-50)$ & $122(16-2,011)$ & $133(22-1,322)$ & $161(0-377)$ & $127(0-2,011)$ \\
\hline Mean \pm SD & $47.2 \pm 3.8$ & $165 \pm 210$ & $227 \pm 255$ & $152 \pm 149$ & $184 \pm 226$ \\
\hline \multicolumn{6}{|l|}{ PDGFR $\alpha$} \\
\hline I & $1.0(1.0)$ & $1.8(2.0)$ & $1.9(2.0)$ & $0.9(1.0)$ & $1.8(2.0)$ \\
\hline $\mathrm{E}$ & $45(45.0)$ & $77(85.0)$ & $83(90.0)$ & $58(70.0)$ & $78(85.0)$ \\
\hline S & $45 \pm 49$ & $150 \pm 88$ & $154 \pm 79$ & $68 \pm 55$ & $148 \pm 85$ \\
\hline \multicolumn{6}{|c|}{ PHH3 per 50 HPFs } \\
\hline Median (range) & $3.5(0-7)$ & $11(0-605)$ & $15(1-219)$ & $7(3-15)$ & $13(0-605)$ \\
\hline Mean \pm SD & $3.5 \pm 4.9$ & $27.3 \pm 77.3$ & $29.6 \pm 42.6$ & $8.3 \pm 6.1$ & $27.6 \pm 66.3$ \\
\hline \multicolumn{6}{|l|}{ P16 } \\
\hline I & $0.5(0.5)$ & $0.6(0.0)$ & $0.9(1.0)$ & $0.7(1.0)$ & $0.7(0.5)$ \\
\hline $\mathrm{E}$ & $20(20.0)$ & $11(0.0)$ & $21(10.0)$ & $15(5.0)$ & $14(2.5)$ \\
\hline $\mathrm{S}$ & $20 \pm 28$ & $17 \pm 38$ & $36 \pm 69$ & $15 \pm 22$ & $23 \pm 50$ \\
\hline \multicolumn{6}{|l|}{ P53 } \\
\hline I & $0.0(0.0)$ & $0.4(0.0)$ & $0.5(0.0)$ & $0.3(0.0)$ & $0.4(0.0)$ \\
\hline $\mathrm{E}$ & $0(0.0)$ & $6(0.0)$ & $11(0.0)$ & $5(0.0)$ & $8(0.0)$ \\
\hline S & $0 \pm 0$ & $7 \pm 15$ & $17 \pm 41$ & $5 \pm 10$ & $10 \pm 27$ \\
\hline \multicolumn{6}{|l|}{$\mathrm{SMA}^{\mathrm{a}}$} \\
\hline I & $1.5(1.5)$ & $0.6(0.0)$ & $1.0(1.0)$ & $0.5(0.5)$ & $0.7(1.0)$ \\
\hline E & $50(50.0)$ & $11(0.0)$ & $23(10.0)$ & $4(2.5)$ & $15(5.0)$ \\
\hline S & $150 \pm 212$ & $18 \pm 43$ & $39 \pm 56$ & $4 \pm 5$ & $26 \pm 52$ \\
\hline
\end{tabular}


Table IV. Continued.

Tumor site

\begin{tabular}{|c|c|c|c|c|c|}
\hline Characteristics & Esophagus & Stomach & Small intestine & Rectum & All sites \\
\hline \multicolumn{6}{|l|}{ S-100 } \\
\hline I & $0.0(0.0)$ & $0.2(0.0)$ & $0.2(0.0)$ & $0.4(0.0)$ & $0.2(0.0)$ \\
\hline $\mathrm{E}$ & $0(0.0)$ & $3(0.0)$ & $3(0.0)$ & $2(0.0)$ & $3(0.0)$ \\
\hline $\mathrm{S}$ & $0 \pm 0$ & $5 \pm 22$ & $6 \pm 38$ & $2 \pm 3$ & $5 \pm 28$ \\
\hline
\end{tabular}

I and $\mathrm{E}$ are expressed as mean (median); $\mathrm{S}$ is expressed as mean $\pm \mathrm{SD}$. ${ }^{\mathrm{S}}$ Significant differences between GIST sub-localizations using analysis of variance. ${ }^{\mathrm{b}}$ Analyzed as previously described (15). GIST, gastrointestinal stromal tumor; I, intensity; E, extent; S, quickscore; HPFs, high-power fields; SD, standard deviation; Bcl-2, B-cell lymphoma 2; DOG-1, discovered on GIST 1; PDGFR $\alpha$, platelet-derived growth factor receptor $\alpha$; PHH3, phosphohistone H3; SMA, smooth muscle actin.

PDGFR $\alpha$ and Bcl-2; P<0.01, ANOVA), TCN (Ki-67, PHH3, desmin, PDGFR $\alpha$, P53 and Bcl-2) and SF (Ki-67, CD-34, PDGFR $\alpha$, P53 and Bcl-2) as well as GP (Ki-67, PHH3, desmin and $\mathrm{P} 53$; $\mathrm{P}<0.05$, t-test). Additionally, Ki-67-based proliferation rate was significantly associated with the histomorphological characteristics of MI and MU in GISTs ( $\mathrm{P}<0.05$, t-test).

Multivariate analysis of clinicopathological, histomorphological and immunohistochemical characteristics according to recurrence, metastasis and overall survival (Table V). The independent variables for multivariate analysis [binary logistic regression (BR) and Cox regression (CR) analysis, summarized in Table $\mathrm{V}$ ] are listed in descending order of their odds ratio (OR).

Recurrence. GP, margin status, localization of the GIST and immunhistochemical CD34 score were time-independent predictors (BR), whereas the same variables (without CD34 score) were identified as time-dependent predictors (CR) for recurrence.

Metastasis. GP was the most significant independent predictor for both mathematical models (BR and CR). H\&E-based mitosis count (per $50 \mathrm{HPFs}$ ) was identified as an additional independent predictor for $\mathrm{BR}$ and maximum size of the GIST was an independent predictor for CR.

Survival. Multifocality, Ki-67-based proliferation rate (per $\mathrm{mm}^{2}$ ) and immunhistochemical CD34 score were identified as independent survival predictors using BR. In comparison, only $\mathrm{Ki}$-67-based proliferation rate (per $\mathrm{mm}^{2}$ ) was an independent factor for survival when applying CR.

Focusing on independent variables with an OR $>5$, only margin status, GP, multifocality and localization of GISTs were identified as independent variables with the highest effect (Table V).

Kaplan-Maier-analyses (Fig. 1). Based on the predicted probabilities, dichotomous variables were calculated to perform further graphical assessment for the prognosis of recurrence, metastasis and survival in GISTs using the Kaplan-Meier method. Based on an optimized cluster analysis, high selective cut-offs for the probability of recurrence (0.1), metastasis (0.05) and survival (0.025) were calculated. The calculated models had the ability to significantly differentiate the risk for all time-to-event procedures for patients with GIST
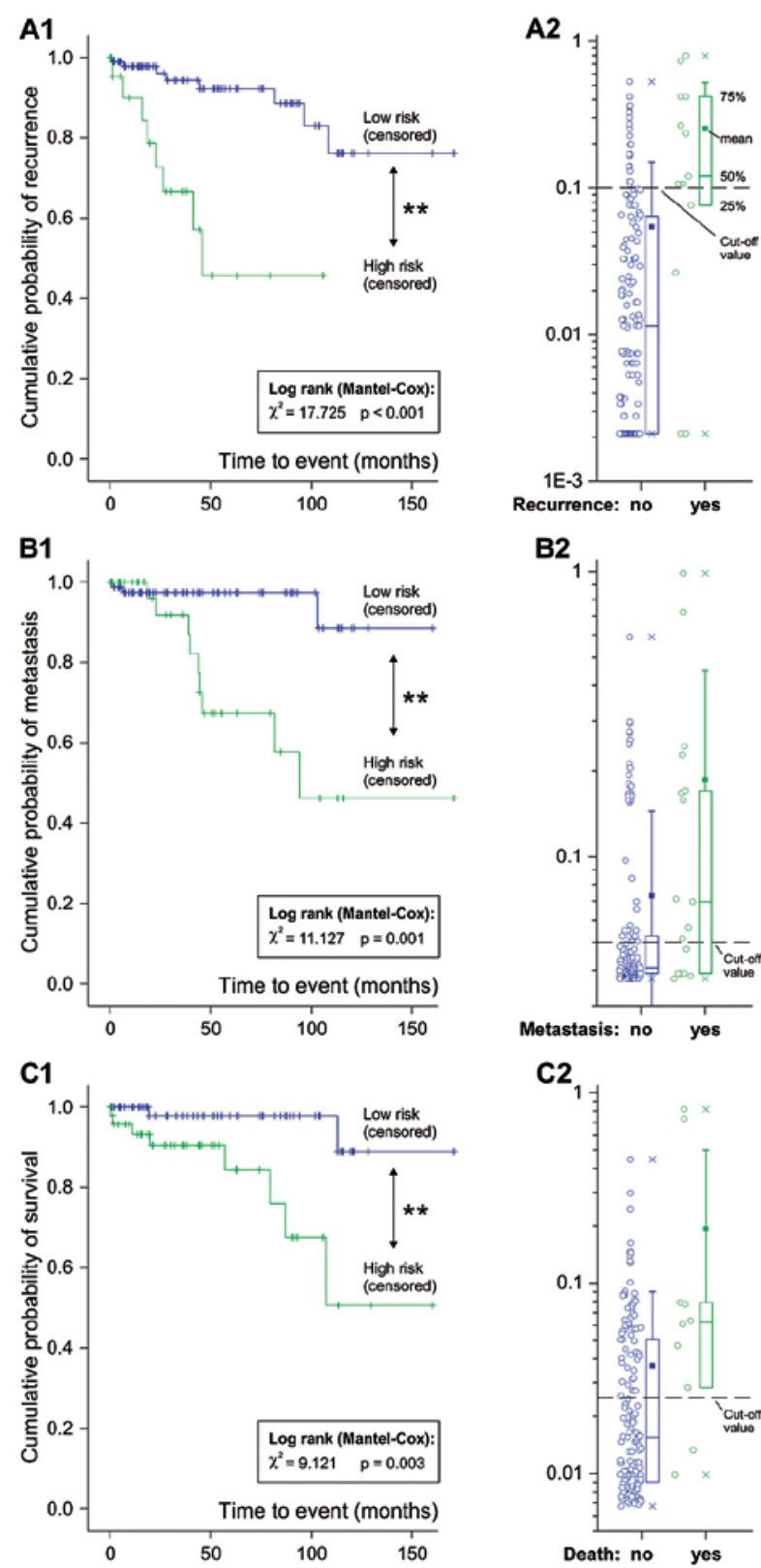

Figure 1. Kaplan-Maier curves for (A1) recurrence, (B1) metastasis and (C1) survival stratified on the predicted probabilities (A2, B2 and C2, respectively) for these events based on the logistic regression analysis. The cut-off values for the predicted probabilities were added to the graphs A1, B1 and C1. 


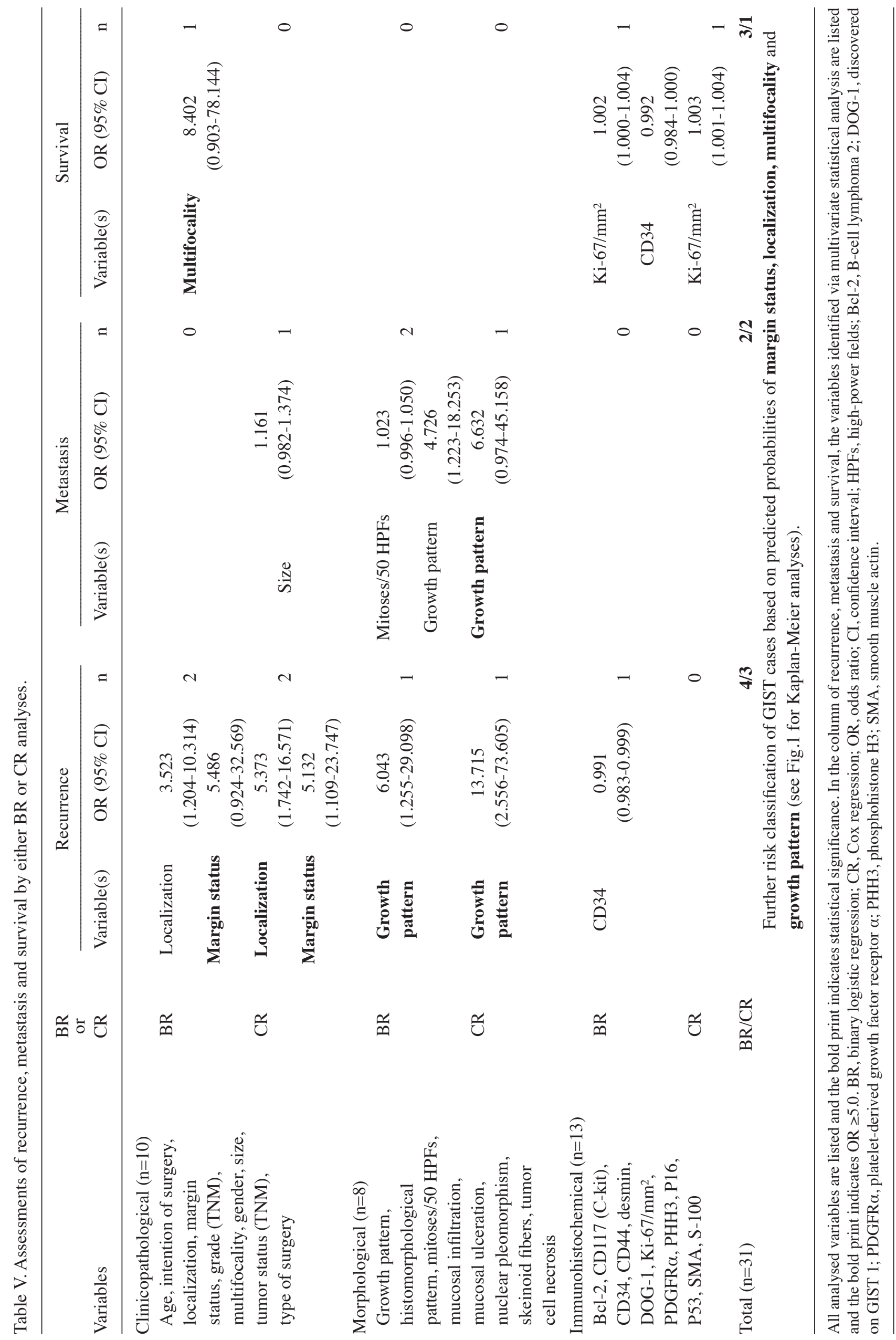


based on the evaluated independent risk factors (Kaplan-Meier method, log-rank test $<0.05)$. Comparing the significance levels of the log-rank tests, the best prognostic model was obtained for recurrence, followed by metastasis and survival. Comparing the graphs, the event recurrence of patients with GIST occurred earlier compared with metastasis or death.

\section{Discussion}

Histomorphological parameters for predicting the biological behavior of GISTs have been analyzed and validated for several years leading to consensual statements. However, it remains difficult to distinguish benign from malignant tumors (3). In our study, we presented a large series of 201 GIST cases with complete clinicopathological evaluation and analyzed these cases for classical histomorphological criteria and immunohistochemical markers using TMAs. The clinical data of age, gender, localization, grading (mitotic activity), as well as surgical procedures and different outcome parameters (recurrence, metastasis and survival) are largely comparable to published GIST series (4), indicating absence of special bias factors.

In the univariate analysis, first of all, a number of variables were associated with recurrence, metastasis and survival, such as NP, TCN and GP (for recurrence and metastasis), CD117 and CD34 scores (for recurrence), CD117 score (for metastasis), and CD34 and PDGFR $\alpha$ scores (for survival). Of note, a considerable amount of the published clinicopathological investigations reveal heterogeneous subsets of prognostic and predictive markers, ranging from morphological parameters, such as gastrointestinal bleeding and tumor size, to molecular parameters, such as expression of SMA, CD34, DOG-1 or CD117, as well as novel markers, such as S-phase kinase-associated protein 2 or carbonic anhydrase II (17-20). However, the majority of these studies, as well as ours, were limited by the fact that most of the statistically significant variables based on univariate statistics are not statistically strong enough to 'survive' a further multivariate analysis, leading to the heretic question of whether IHC does actually provide additional prognostic data in GISTs (18). Furthermore, we were unable to confirm the role of differentiation and cell-cycle regulatory proteins, such as Bcl-2, P16 and P53, as well as CD44, as prognostic factors, similar to previous studies $(8,21-24)$. The reasons may be different antibodies, antigen retrieval or inter-observer variations of the immunohistochemical staining $(10,11,15)$.

In the multivariate analysis, the number of variables in a time-independent/time-dependent analysis was significantly reduced from a total of $31(100 \%)$ to $4(12.9 \%) / 3(9.6 \%)$ for recurrence, $2(6.4 \%) / 2(6.4 \%)$ for metastasis and $3(9.6 \%) / 1(3.2 \%)$ for survival (Table V), indicating the rarity of suitable endpoint markers available for GISTs to date. Interestingly, classical and 'conservative'/“well-established' parameters, such as tumor localization, margin status, multifocality, as well as H\&E-based mitosis and Ki-67-based proliferation rate of GISTs, were significantly associated with defined clinical endpoints, as already described by the European Sarcoma Network Working Group in 2012 (25). This, however, confirms earlier clinical studies $(26,27)$ and indicates that powerful prognostic molecular markers (with the exception of CD34) are still not available to date. Of note, in contrast to the findings of Demir et al (18), lower immunhistochemical CD34 score was associated with higher recurrence rate and shorter survival, whereas this significant effect of CD34 expression was lost when performing a multivariate Cox regression analysis.

Additionally, a 'new' and prognosis-relevant parameter as reported by Agaimy et al (7) is GP, namely type I (luminal/intramural) or type II (extramural), which was found to be an independent prognostic factor for recurrence and metastasis (with an OR of $\leq 13.715$ for recurrence). Therefore, preoperative radiological analysis and GP evaluation is essential for the surgical strategies of local or extensive resection. Furthermore, GP should be macroscopically described in detail by the surgeons and pathologists and should be included in further tumor stratifications of GISTs, such as the classical risk stratification scheme of Miettinen et al and others (2,28-30).

Comparing the current uni- and multivariate statistical analyses, it becomes evident that more parameters may be associated with the clinicopathological endpoints (recurrence, metastasis and survival) in the univariate rather than in the multivariate statistical analysis. This is clearly caused by the applied statistical approaches of excluding variables using ANOVA with forward WALD procedures. Therefore, the statistical power of the univariate analysis is very limited, as it was similarly found in most previous GIST studies on prognostic makers, whereas all these mathematical procedures may include bias to disregard the biological impact of possible predictive variables (31). However, we were able to calculate statistically significant models based on the predicted probabilities of the regression analysis to provide prognostic information for recurrence, metastasis and survival of patients with GISTs. Such an approach may be helpful in developing such 'heat maps', as reported by Joensuu et al, to provide clinicians and patients with better prognostic information for relevant endpoints (32).

In summary, our investigations demonstrated that i) classical parameters of GISTs, such as localization, multifocality and H\&E-based mitosis/Ki-67-based proliferation, have the most relevant prognostic value; ii) GP of GISTs must be taken into consideration as new prognostic factor and further validated; and iii) there remains a need for efficient molecular markers for GISTs.

\section{Acknowledgements}

The expert technical assistance of Mrs. Berta Lechner, Mrs. Monika Prinz, Mrs. Ines Grob-Achleitner and Mr. Brian van Merkestijn is gratefully acknowledged. The present study was supported by the Wissenschaftlicher Verein der Pathologie Salzburg/Austria.

\section{References}

1. Nilsson B, Bümming P, Meis-Kindblom JM, Odén A, Dortok A, Gustavsson B, Sablinska K and Kindblom LG: Gastrointestinal stromal tumors: The incidence, prevalence, clinical course, and prognostication in the preimatinib mesylate era - a population-based study in western Sweden. Cancer 103: 821-829, 2005.

2. Miettinen M and Lasota J: Gastrointestinal stromal tumors: Pathology and prognosis at different sites. Semin Diagn Pathol 23: 70-83, 2006.

3. Fletcher CD, Berman JJ, Corless C, Gorstein F, Lasota J, Longley BJ, Miettinen M, O'Leary TJ, Remotti H, Rubin BP, et al: Diagnosis of gastrointestinal stromal tumors: A consensus approach. Hum Pathol 33: 459-465, 2002. 
4. Sorour MA, Kassem MI, Ghazal Ael-H, El-Riwini MT and Abu Nasr A: Gastrointestinal stromal tumors (GIST) related emergencies. Int J Surg 12: 269-280, 2014.

5. Miettinen M, El Rifai W, H L Sobin L and Lasota J: Evaluation of malignancy and prognosis of gastrointestinal stromal tumors: A review. Hum Pathol 33: 478-483, 2002.

6. Rossi S, Miceli R, Messerini L, Bearzi I, Mazzoleni G, Capella C Arrigoni G, Sonzogni A, Sidoni A, Toffolatti L, et al: Natural history of imatinib-naive GISTs: A retrospective analysis of 929 cases with long-term follow-up and development of a survival nomogram based on mitotic index and size as continuous variables. Am J Surg Pathol 35: 1646-1656, 2011.

7. Agaimy A, Vassos N, Wunsch PH, Hohenberger W, Hartmann A and Croner RS: Impact of serosal involvement/extramural growth on the risk of synchronous and metachronous peritoneal spread in gastrointestinal stromal tumors: Proposal for a macroscopic classification of GIST. Int J Clin Exp Pathol 5: 12-22, 2012.

8. Panizo-Santos A, Sola I, Vega F, de Alava E, Lozano MD, Idoate MA and Pardo-Mindán J: Predicting metastatic risk of gastrointestinal stromal tumors: Role of cell proliferation and cell cycle regulatory proteins. Int J Surg Pathol 8: 133-144, 2000.

9. Tannapfel A and Wittekind C: The current TNM system for gastrointestinal tumors part II. Pathologe 31: 348-352, 2010 (In German)

10. Illig R, Fritsch $\mathrm{H}$ and Schwarzer $\mathrm{C}$ : Breaking the seals: Efficient mRNA detection from human archival paraffin-embedded tissue. RNA: 15: 1588-1596, 2009.

11. Illig R, Fritsch H and Schwarzer C: Spatio-temporal expression of HOX genes in human hindgut development. Dev Dyn 242: 53-66, 2013.

12. Brainard JA and Goldblum JR: Stromal tumors of the jejunum and ileum: A clinicopathologic study of 39 cases. Am J Surg Pathol 21: 407-416, 1997.

13. Kemmerling R, Stintzing S, Mühlmann J, Dietze $O$ and Neureiter D: Primary testicular lymphoma: A strictly homogeneous hematological disease? Oncol Rep 23: 1261-1267, 2010.

14. Kemmerling R, Alinger B, Dietze O, Bösmüller HC, Ocker M, Wolkersdörfer GW, Berr F, Neureiter D and Kiesslich T: Association of stem cell marker expression pattern and survival in human biliary tract cancer. Int J Oncol 41: 511-522, 2012.

15. Kemmerling R, Weyland D, Kiesslich T, Illig R, Klieser E, Jäger T, Dietze O and Neureiter D: Robust linear regression model of Ki-67 for mitotic rate in gastrointestinal stromal tumors. Oncol Lett 7: 745-749, 2014.

16. Kiesslich T, Alinger B, Wolkersdörfer GW, Ocker M, Neureiter D and Berr F: Active Wnt signalling is associated with low differentiation and high proliferation in human biliary tract cancer in vitro and in vivo and is sensitive to pharmacological inhibition. Int J Oncol 36: 49-58, 2010

17. Lv A, Li Z, Tian X, Guan X, Zhao M, Dong B and Hao C: SKP2 high expression, KIT exon 11 deletions, and gastrointestinal bleeding as predictors of poor prognosis in primary gastrointestinal stromal tumors. PLoS One 8: e62951, 2013.

18. Demir L, Ekinci N, Erten C, Kucukzeybek Y, Alacacioglu A, Somali I, Can A, Dirican A, Bayoglu V, Akyol M, et al: Does immunohistochemistry provide additional prognostic data in gastrointestinal stromal tumors? Asian Pac J Cancer Prev 14 4751-4758, 2013
19. Liu LC, Xu WT, Wu X, Zhao P,Lv YL and Chen L: Overexpression of carbonic anhydrase II and Ki-67 proteins in prognosis of gastrointestinal stromal tumors. World J Gastroenterol 19: 2473-2480, 2013

20. Qi Y, Zhao W, Wang Z, Li T and Meng X: Tumor sites and microscopic indicators are independent prognosis predictors of gastrointestinal stromal tumors. Tohoku J Exp Med 233: 65-72, 2014.

21. Rudolph P, Gloeckner K, Parwaresch R, Harms D and Schmidt D: Immunophenotype, proliferation, DNA ploidy, and biological behavior of gastrointestinal stromal tumors: A multivariate clinicopathologic study. Hum Pathol 29: 791-800, 1998.

22. Gumurdulu D, Erdogan S, Kayaselcuk F, Seydaoglu G, Parsak CK Demircan O and Tuncer I: Expression of COX-2, PCNA, Ki-67 and p53 in gastrointestinal stromal tumors and its relationship with histopathological parameters. World J Gastroenterol 13: 426-431, 2007.

23. Fujita A, Yamamoto H, Imamura M, Nakamura N, Maehara Y, Tsuneyoshi M and Oda Y: Expression level of the mitotic checkpoint protein and G2-M cell cycle regulators and prognosis in gastrointestinal stromal tumors in the stomach. Virchows Arch 460: 163-169, 2012.

24. Meara RS, Cangiarella J, Simsir A, Horton D, Eltoum I and Chhieng DC: Prediction of aggressiveness of gastrointestinal stromal tumours based on immunostaining with bcl-2, Ki-67 and p53. Cytopathology 18: 283-289, 2007.

25. ESMO/European Sarcoma Network Working Group: Gastrointestinal stromal tumors: ESMO Clinical Practice Guidelines for diagnosis, treatment and follow-up. Ann Oncol 23 (Suppl 7): vii49-vii55, 2012.

26. Emory TS, Sobin LH, Lukes L, Lee DH and O'Leary TJ: Prognosis of gastrointestinal smooth-muscle (stromal) tumors: Dependence on anatomic site. Am J Surg Pathol 23: 82-87, 1999.

27. Yan H, Marchettini P, Acherman YI, Gething SA, Brun E and Sugarbaker PH: Prognostic assessment of gastrointestinal stromal tumor. Am J Clin Oncol 26: 221-228, 2003.

28. Miettinen $\mathrm{M}$ and Lasota J: Gastrointestinal stromal tumors: Review on morphology, molecular pathology, prognosis and differential diagnosis. Arch Pathol Lab Med 130: 1466-1478, 2006.

29. Miettinen M and Lasota J: Histopathology of gastrointestinal stromal tumor. J Surg Oncol 104: 865-873, 2011.

30. Reichardt P and Reichardt A: Gastrointestinal stromal tumors (GIST). Dtsch Med Wochenschr 138: 2013-2016, 2013 (In German).

31. Lovell DP: Biological importance and statistical significance. J Agric Food Chem 61: 8340-8348, 2013.

32. Joensuu H, Vehtari A, Riihimäki J, Nishida T, Steigen SE, Brabec P, Plank L, Nilsson B, Cirilli C, Braconi C, et al: Risk of recurrence of gastrointestinal stromal tumour after surgery: An analysis of pooled population-based cohorts. Lancet Oncol 13: 265-274, 2012. 